\title{
Calidad del manejo de pacientes con insuficiencia cardiaca en el Servicio de Medicina Interna
}

\author{
M. T. ÁLVAREZ FRÍAS, J. GUTIÉRREZ DUBOIS J. L. ALONSO MARTÍNEZ, \\ M. SOLANO REMÍREZ, C. GONZÁLEZ ARENCIBIA, M. ETXEGARAY AGARA
}

Servicio de Medicina Interna. Hospital de Navarra. Pamplona

\author{
QUALITY MANAGEMENT IN PATIENTS WITH HEART FAILURE \\ INSIDE AN INTERNAL MEDICINE DEPARTMENT
}

\section{RESUMEN}

Objetivo: Evaluar la calidad asistencial de los pacientes ingresados en nuestro Servicio de Medicina Interna con diagnóstico de insuficiencia cardiaca, basándonos en una serie de criterios de calidad recogidos en el estudio ACOVE.

Métodos: Análisis retrospectivo mediante protocolo de datos de 267 informes de alta de pacientes ingresados en Medicina Interna en los que uno de los diagnósticos fue el de insuficiencia cardiaca (periodo de enero 2001- enero 2002).

Resultados: Valoramos 267 informes de alta (edad media $76 \pm 9$, siendo varones el 50\%). Respecto al estudio ACOVE la puntuación media obtenida fue de $6,72 \pm 1,33$. Se determinó las causas de insuficiencia cardiaca en el $82 \%$ de los casos (33\% isquémico-dilatada, 30\% hipertensiva, $12 \%$ valvular y $7 \%$ otras). El empleo de IECAS/ARA II se realizó en el $66 \%$ de los pacientes, con escasa utilización de otros fármacos como los $\beta$-bloqueantes (16\%), calcioantagonistas (7\%) y antiarrítmicos de clase I (1\%). El 94\% de los pacientes recibieron instrucciones breves acerca del manejo de su enfermedad. Sólo un $36 \%$ presentaban estudio ecocardiográfico. El los pacientes con fibrilación auricular, el 19\% fueron tratados con anticoagulación y el $26 \%$ con antiagregación. La mortalidad intrahospitalaria fue de un $4 \%$. En el estudio, no hubo diferencias de puntuación entre los diferentes staff del departamento en el manejo de la insuficiencia cardiaca. Así mismo, tampoco se hallaron diferencias en relación al sexo. La edad fue un factor a tener en cuenta: $>70$ años, score $6,5 \pm 1,38 ;<70$ años, score $7,15 \pm 1,17(\mathrm{p}=0,011)$.

Conclusiones: El manejo de la insuficiencia cardiaca en nuestro servicio de M.Interna es aceptable. Sin embargo, existen importantes puntos donde se debería mejorar, como el aumento de la utilización de IECAS y b-bloqueantes en la insuficiencia cardiaca y el incremento en el uso de la ecocardiografía. Además, en los pacientes de edad avanzada se ha demostrado que el nivel de calidad alcanzado es inferior, lo cual deberemos mejorar.

PALABRAS CLAVE: Insuficiencia cardiaca. Calidad asistencial. Indicadores de calidad (ACOVE).

ABSTRACT

Objective: To evaluate the quality management of Heart failure within an Internal Medicine Department, based in quality criteria settled in ACOVE study.

Methods: Retrospective study reporting 267 patients admitted to our Internal Medicine Department with a diagnosis of heart failure (from January 2001 to January 2001). We applied ACOVE protocol to evaluate quality of management assigning a positive numerical score to every accomplished section and a negative score to those sections that were not carried out.

Results: Two hundred and sixty seven patients and their clinical records were evaluated (Mean age $76 \pm 9$ years, male 50\%). They had a mean score of $6.72 \pm 1.33$ points.

Heart failure etiology was determined in $82 \%$ (33\% ischemic heart failure, $30 \%$ hypertensive heart disease, $12 \%$ valvulopathy and $7 \%$ others). ACE-Inhibitors/ARA II were used in $66 \%$ of patients, with poor utilization of beta-blockers (16\%), calcium channel blockers (7\%) and class I antiarrhythmic drugs (1\%). $94 \%$ of patients had written instructions about manage of their disease. Only $36 \%$ of patients had an echocardiography study. In patients with atrial fibrillation, $19 \%$ were treated with oral anticoagulants and $26 \%$ with anti-platelet drugs. Inhospital mortality rate was $4 \%$

We could not meet differences among different physicians and their gender in department of Internal Medicine treating for heart failure, however the score of patients older 70 years was $6.5 \pm 1.38$ points while score in younger to years was $7.15 \pm 1.17$ points $(p=0.011)$.

Conclusions: Management of heart failure in our department of Internal Medicine is acceptable. However, there are several points in which improvement could be reached, much as to increase the utilization of ACE inhibitors and beta-blockers in handling of heart failure and to rise the are of echocardiography in the evaluation of these patiens. Moreover, older patients showed a lower quality level that could be improved.

KEY WORDS: Heart failure. Quality management. Quality indicators (ACOVE).

Álvarez Frías MT, Gutiérrez Dubois J, Alonso Martínez JL, Solano Remírez M, González Arencibia C, Etxegaray Agara M. Calidad del manejo de pacientes con insuficiencia cardiaca en el Servicio de Medicina Interna. An Med Interna (Madrid) 2005; 22: 309-312. 


\section{INTRODUCCIÓN}

La insuficiencia cardiaca es un importante problema de salud y tanto la prevalencia como la incidencia de este problema sigue aumentando en los países desarrollados a diferencia de otras enfermedades cardiovasculares que van siendo controladas $(1,2)$. Este trastorno ocasiona un alto grado de mortalidad, gran número de ingresos hospitalarios, alto consumo de recursos sanitarios y disminución en la calidad de vida (3). En España, los médicos internistas tratan a muchos pacientes con insuficiencia cardiaca $(4,5)$, y los enfermos hospitalizados en Medicina Interna difieren de los ingresados en Servicios de Cardiología, siendo principalmente mujeres ancianas, con fracción de eyección conservada, bajo nivel cultural y alta dependencia física (6). En nuestro país se han llevado a cabo algunos estudios de calidad en la insuficiencia cardiaca que demuestran en general un inadecuado manejo del paciente con insuficiencia cardiaca $(7,8)$. Se han comunicado estándares de calidad del manejo de la insuficiencia cardiaca (9). Nuestro objetivo fue el conocer el grado de ajuste a un estándar de calidad de los pacientes ingresados en nuestro servicio por insuficiencia cardiaca.

\section{MATERIAL Y MÉTODOS}

Estudiamos de forma retrospectiva a todos los pacientes en cuyo informe de alta constara el diagnóstico de insuficiencia cardiaca, bien como primer diagnóstico o como diagnóstico secundario, en el periodo de enero 2001 a enero 2002. Los informes de alta de hospitalización fueron entregados al alta a cada paciente, constando ineludiblemente de antecedentes personales, historia actual, anamnesis por aparatos, exploraciones complementarias y tratamiento a seguir. El epígrafe del tratamiento incluía la dieta recomendada, régimen de vida y tratamiento farmacológico. Nos basamos en el estudio ACOVE (9) para valorar la calidad de los cuidados en la insuficiencia cardiaca (Tabla I).

Aplicamos puntuación positiva a la recogida de ciertos datos en la historia clínica (presencia o ausencia de infarto agudo de miocardio, enfermedad coronaria, revascularización, hipertensión arterial, dolor torácico, hábito tabáquico, enfermedad tiroidea, calasificación NYHA, tratamiento actual), a la realización de una exploración física completa (peso corporal, tensión arterial, frecuencia cardiaca, auscultación cardio-pulmonar, exploración abdominal y de extremidades inferiores), y de exploraciones complementarias ( $\mathrm{Rx}$ tórax, ECG, monitorización de iones, determinación de TSH en los pacientes indicados), a ser tratados durante el ingreso o al alta con IECAS o ARA II y/o betabloqueantes, tener monitorización de iones durante el ingreso, la realización de ecocardiograma con medición de la fracción de eyección del ventrículo izquierdo tres meses antes o después del ingreso, la medición de la digoxinemia en pacientes que la tomaban y estuvieran en fibrilación auricular (FA), la anticoagulación al alta de pacientes con FA y la instrucción del paciente o su remisión al médico de atención primaria para control si empeoraba. Con la mitad de la puntuación positiva se calificó a los pacientes con FA y antiagregación, y con puntuación negativa a aquellos pacientes que al alta estaban tratados con calcioantagonistas que no fueran de $3^{\text {a }}$ generación y/o con antarrítmicos de clase I. La máxima puntuación posible a alcanzar era de 10 puntos.

\section{TABLA I}

\section{INDICADORES DE CALIDAD EN EL MANEJO DE LA INSUFICIENCIA CARDIACA (ACOVE)}

\begin{tabular}{|c|c|}
\hline Indicador 1 & $\begin{array}{l}\text { IECA en el tratamiento de la disfunción ventricular izq asinto- } \\
\text { mática }\end{array}$ \\
\hline Indicador 2 & IECA en la enfermedad cardíaca sintomática \\
\hline Indicador 3-4 & Datos de la historia clínica (1) y exploración física (2) \\
\hline Indicador 5 & Pruebas diagnósticas realizadas (3) \\
\hline Indicador 6 & $\begin{array}{l}\text { Educación del paciente acerca de la enfermedad y del manejo } \\
\text { terapeútico }\end{array}$ \\
\hline Indicador 7 & Evaluación de la fracción de eyección del VI \\
\hline Indicador 8 & Monitorización bioquímica durante la hospitalización (4) \\
\hline Indicador 9 & B-bloqueantes en la enfermedad cardíaca \\
\hline Indicador 10 & Empleo de calcioantagonistas en la enfermedad cardiaca \\
\hline Indicador 11 & Antiarrítmicos tipo I en la enfermedad cardiaca \\
\hline Indicador 12 & Monitorización de niveles de digoxinemia \\
\hline Indicador 13-14 & Diagnóstico y manejo de la fibrilación auricular \\
\hline
\end{tabular}

1. Presencia o ausencia de IAM previo, enfermedad coronaria, revascularización, dolor torácico y angina, historia de HTA, DM o enfermedad tiroidea, tabaquismo, trtamiento actual y capacidad actual (NYHA).

2. Peso corporal, TA, frecuencia cardiaca, datos de exploración pulmonar, cardiaca, abdominal y EEll.

3. En el plazo de un mes; Rx tórax, ECG, hemograma, ionograma, creatinina sérica, TSH (en pacientes con FA o con insuficiencia cardiaca sin causa conocida).

4. Electrolitos, creatinina y urea.

Análisis estadístico; las variables continuas son expresadas en medias ( \pm desviación estándar). Las variables categóricas son expresadas en porcentajes. Se empleó la prueba de la " $t$ " de Student para comparar medias de variables continuas con distribución normal, y la prueba de la "U" de Mann Whitney cuando presentaba distribución no paramétrica. Analizamos la correlación entre variables continuas con la prueba de Pearson cuando teníamos una distribución normal, y la prueba de Sperman para distribuciones no paramétricas. Así mismo, analizamos la influencia de los años de ejercicio profesional sobre la puntuación basada en el estudio ACOVE mediante ANOVA. Las diferencias se consideraron significativas cuando se obtuvo un valor de $\mathrm{p}<0,05$.

\section{RESULTADOS}

Se evaluaron en el estudio 267 informes de alta de pacientes ingresados en Medicina Interna en los que uno de los diagnósticos fue el de insuficiencia cardiaca. La edad media de los pacientes ingresados fue de $76 \pm 9$ años de los que el $50 \%$ resultaron mujeres.

La duración media del ingreso hospitalario fue de $9 \pm 5$ días, con un rango que variaba desde 1-27 días, no existiendo correlación entre la estancia media, la causa de la insuficiencia cardiaca y la edad. Así mismo, la mortalidad durante el ingreso hospitalario fue del $4 \%$.

Las causas más frecuentes de insuficiencia cardiaca fueron la cardiopatía isquémico en fase dilatada (33\%), la cardiopatía hipertensiva (30\%), la cardiopatía valvular (12\%), y miscelánea $(7 \%)$. La insuficiencia cardiaca de causa no filiada representó un 18\% (Fig. 1). Los desencadenantes de la descompensación de la insuficiencia cardiaca fueron la infección 

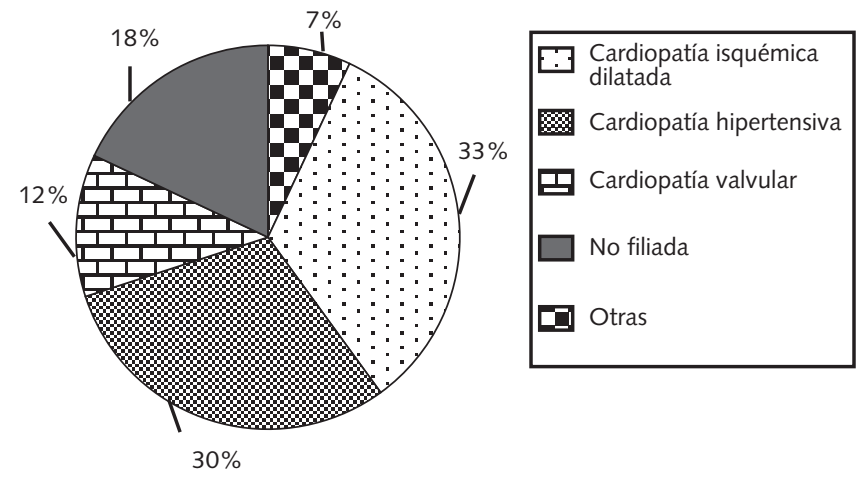

Fig. 1. Causas de insuficiencia cardiaca.

respiratoria (24\%), la anemia (12\%), la toma de AINES (7\%), otras $(7 \%)$, no recogiéndose este dato en el $50 \%$ de los casos (Fig. 2).

Con respecto a los indicadores de calidad ACOVE la puntuación media obtenida por cada paciente fue de $6.72 \pm 1.33$ (r 2.1-9.6). Cada uno de los ítems quedan desglosados como se aprecia en la tabla II.

Indicador 1-2: El 64\% de los pacientes con disfunción ventricular izquierda recibieron tratamiento con IECA/ARA II. Indicador 3: Prácticamente la totalidad de los pacientes estudiados cumplían estos indicadores (antecedentes de IAM $18 \%$, enfermedad coronaria $16 \%$, revascularización $10 \%$, dolor torácico $80 \%$, HTA $73 \%$, enfermedad tiroidea $56 \%$, hábito tabáquico 76\%, clasificación de la NYHA 30.5\%, tra-

\section{TABLA II}

RESULTADOS RECOGIDOS EN EL ESTUDIO REALIZADO EN NUESTRO SERVICIO DE MEDICINA INTERNA

Indicadores 1-2 El 64\% de los pacientes recibieron tratamiento con IECAS/ARA ||

Indicador 3 IAM (18\%), enfermedad coronaria (16\%), revascularización (10\%), dolor torácico (80\%), HTA (73\%), enfermedad tiroidea $(56 \%)$, hábito tabáquico $(76 \%)$, clasificación de NYHA (30.5\%), tratamiento actual (94\%)

Indicador $4 \quad$ Peso corporal (27\%), TA (97\%), frecuencia cardíaca (75\%), auscultación cardio-pulmonar y exploración abdominal (100\%), exploración EEII (99\%)

Indicador $5 \quad$ Rx tórax (99\%), ECG (98\%), monitorización iones (79\%), determinación TSH en los pacientes indicados (41\%)

Indicador $6 \quad$ Se instruía al paciente o se remitía al MAP (94\%)

Indicador $7 \quad$ Realizado dentro de los 3 meses ants y/o posteriores ecocardiografía (36\%)

Indicador $8 \quad$ Hemograma, iones y creatinina (99\%)

Indicador 9 Tratamiento con b-bloqueantes (18\%)

Indicador 10 Tratamiento con calcioantagonistas (7.14\%)

Indicador 11 Tratamiento con antiarrítmicos clase I (0.99\%)

Indicador 12 Determinación de digoxinemia (17\%)

Indicadores 13-14 FA (55\%). El 26\% fueron antiagregados y el 19\% anticoagulados

IAM: infarto agudo de miocardio. HTA: hipertensión arterial. EEII: extremidades inferiores. TSH: hormona tirotropina. MAP: médico de atención primaria. FA: fibrilación auricular.
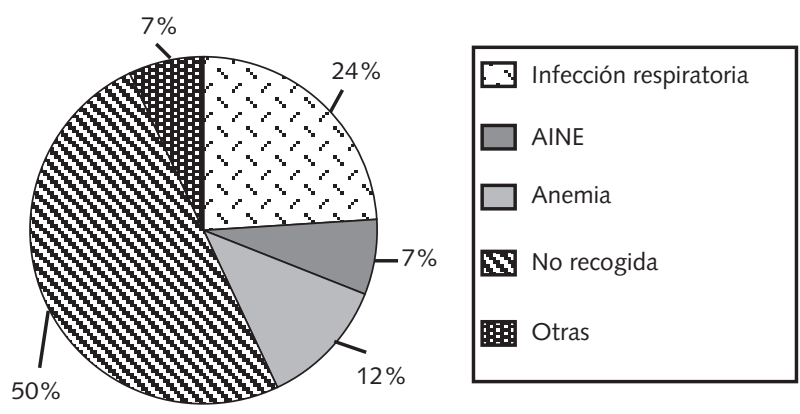

Fig. 2. Desencadenantes de insuficiencia cardiaca.

tamiento actual 94\%). Indicador 4: En la gran mayoría de los pacientes se realizó una exploración física detallada (peso corporal $27 \%$, tensión arterial 97\%, frecuencia cardiaca $75 \%$, auscultación cardiopulmonar y exploración abdominal $100 \%$ y exploración de extremidades inferiores 99\%). Indicador 5: En estos pacientes, en el plazo de un mes entorno al ingreso, se realizó radiología simple de tórax (99\%) y electrocardiograma (98\%). De la misma manera, se monitorizó el hemograma, ionograma y creatinina sérica en el $99 \%$ de los pacientes (con determinación de TSH en pacientes seleccionados en el $41 \%$ de los casos). Indicador 6: De cara al alta hospitalaria, se le instruyó al paciente o al médico de atención primaria a modificar la medicación en caso de empeoramiento clínico en el $94 \%$ de las situaciones. Indicador 7: En cuanto al estudio ecocardiográfico, se realizó valoración de la fracción de eyección en el espacio de tres meses antes y/o después del ingreso en el 36\% de los pacientes. Indicador 8: En el 99\% de los casos se realizó durante el ingreso la determinación de hemograma, iones y creatinina sérica. Indicador 9: Así mismo, se inició tratamiento betabloqueante (en presencia de FE menor de 40 o NYHA I-III) en el 18\% de los pacientes. Indicador 10: Se empleó tratamiento con calcioantagonistas (no $3^{\mathrm{a}}$ generación) en el $7.14 \%$ de los casos. Indicador 11: Y toma de fármacos antiarrítmicos clase I en el 0,99\%. Indicador 12: La determinación de digoxinemia durante el periodo de ingreso se realizó en el $17 \%$ de los pacientes. Indicador 13-14: se objetivó en el 55\% de los casos presencia de fibrilación auricular, realizándose tratamiento antiagregante en el $26 \%$ y tratamiento anticoagulante en el $19 \%$ de los enfermos.

\section{DISCUSIÓN}

Los pacientes que ingresan en los servicios de Medicina Interna son cada vez de mayor edad y la insuficiencia cardiaca sigue siendo la causa más frecuente de ingreso hospitalario en personas mayores de 65 años $(1,3)$. En nuestro servicio, estas características no son diferentes del resto de estudios publicados hasta el momento, constituyendo el $20 \%$ de ingresos anuales y siendo la edad media de los pacientes ingresados de 76 años. Además, con el aumento de la edad aumenta también la complejidad de los pacientes, siendo la mayoría de ellos pluripatológicos, por lo que su manejo se dificulta (7). 
El objetivo de nuestro estudio ha sido evaluar la calidad asistencial de los pacientes ingresados en nuestro servicio con diagnóstico de insuficiencia cardiaca, basándonos en una serie de criterios de calidad recogidos en el estudio ACOVE (9).

El nivel de calidad del manejo alcanzado en nuestro servicio supone aproximadamente el $70 \%$ del ideal, siendo independiente de la edad, sexo y etiología de la insuficiencia cardiaca. La mortalidad intrahospitalaria sólo fue del $4 \%$, algo menor que las publicadas en otros estudios similares, aunque en este sentido hay que reseñar que, en nuestro centro, los pacientes más graves son ingresados generalmente en la Unidad coronaria o en la UCI. Factores que favorecen estos buenos resultados son la realización de una exploración física completa, pruebas complementarias básicas y analítica en practicamente el $100 \%$ de los pacientes, la escasa utilización de fármacos poco indicados como los calcioantagonistas y/o los antiarrítmicos de clase I (7 y $1 \%$ respectivamente), así como el aceptable empleo de IECAS / ARA II (fármacos que han demostrado sobradamente su indicación en este tipo de patología), que en nuestro caso alcanzaba al $66 \%$ de los pacientes, levemente mayor que cifras de estudios similares publicados hasta la fecha (4,8) (Fig. 3). También destaca positivamente el hecho de que al $94 \%$ de los pacientes se les daba instrucciones breves para el manejo de su enfermedad, alimentación y estilo de vida, o se derivaba a su médico de atención primaria para el control de su enfermedad, factores que favorecen un mayor control de este tipo de enfermos.

Sin embargo, también hay puntos donde debemos mejorar. El Ecocardiograma se ha convertido en una exploración imprescindible para el conocimiento de la causa de la insuficiencia cardiaca (6), así como para conocer la situación funcional del

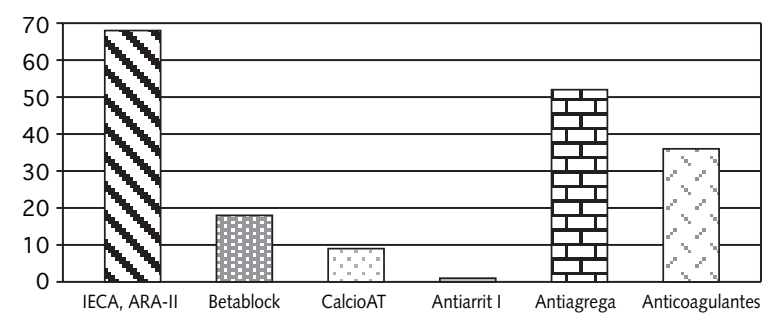

IECA, ARA-II Q Betablock .

Fig. 3.Tratamiento de la insufiencia cardiaca.

\section{Bibliografía}

1. Rodríguez-Artalejo F, Guallar-Castillón P, Banegas JR, Del Rey J. Trends in hospitalization and mortality for heart falilure in Spain, 19801993. Eur Heart J 1997; 18: 1771-1779.

2. Konstam, Marvin A. Heart Failure: Management of patientes with leftventricular systolic dysfunction. Quick Reference Guide for Clinicians. Clinical Practice Guideline No.11. Rockville, MD: V.S. Department of Health and Human Services; 1994. AHCPR publication no. 94-0612.

3. Remme WJ, Swedberg K. Task Force for the Diagnosis and treatment of Chronic Heart Failure, European Society of Cardiology (Co-Chairmen). Eur Heart J 1995; 16: 741-51.

4. Conthe P, Pacho E. El tratamiento de la insuficiencia cardiaca. Rev Clínica Española 2000; 10: 551-62.

5. Encuesta Nacional de la Sociedad Española de Medicina Interna sobre insuficiencia cardiaca. Rev Clin Esp 2001; 201 (Supl. 1): 3-6.

6. Grupo de trabajo de insuficiencia cardiaca de la Sociedad Española de paciente en base a la FE. En nuestro caso, esta exploración no se realizó en todos los casos indicados fundamentalmente por el retraso en el acceso del internista a dicha prueba diagnóstica.

Esto plantearía la cuestión de si sería conveniente el aprendizaje por parte del internista de una modalidad básica de ecocardiografía. El tratamiento con beta bloqueantes en la fase estable de la enfermedad ha demostrado la reducción de la mortalidad así como la hospitalización por insuficiencia cardiaca $(2,9)$. Pese a ello, son fármacos escasamente utilizados muchas veces motivado por la aparición de efectos secundarios como la hipotensión arterial y/o la bradicardia, lo que provoca su retirada. En nuestro caso, solo el $16 \%$ de los pacientes fueron tratados con dichos fármacos. Quizás, el inicio progresivo y a dosis bajas haga que esta cifra pueda ser ampliada en un futuro.

El tratamiento con digoxina en pacientes con insuficiencia cardiaca y arritmia cardiaca por FA está claramente establecido, siendo más discutido en casos de ritmo sinusal. Además, en un estudio reciente, se ha demostrado la diferente acción de la digoxina dependiendo del sexo, así como la mayor tendencia de las mujeres a la intoxicación digitálica. En este sentido la determinación de las cifras de digoxinemia podría evitar las compliaciones asociadas a este fármaco. En nuestro caso, solo en el $17 \%$ de los pacientes que la tomaban se realizó la determinación. Por último, la entrada en FA es un factor de riesgo para la aparición de fenómenos embólicos por lo que está recomendada la anticoagulación siempre y cuando se cumplan una serie de requisitos (edad, situación social y funcional, patología asociada). En nuestro caso, el $19 \%$ de los pacientes estaban anticoagulados y el $26 \%$ antiagregados, cifras que si bien son escasas, podrían ser debidas a que los pacientes ingresados son de edad avanzada y su situación social junto con su patología asociada, no ayuda a la correcta cumplimentación del tratamiento anticoagulante.

En conclusión, podemos afirmar que la revisión de los informes de alta de los pacientes diagnosticados de insuficiencia cardiaca, basándonos en criterios de calidad reconocidos en el estudio ACOVE, pueden ser una herramienta muy útil para conocer la realidad de la calidad del manejo de estos pacientes dentro de un servicio de M Interna. Sin embargo, hay que señalar que son necesarios estudios más amplios que impliquen no sólo a la asistencia hospitalaria sino además, el seguimiento de los enfermos por parte de Atención primaria, para así conocer si el tratamiento y manejo de estos pacientes es el óptimo (10).
Medicina Interna (SEMI). La insuficiencia cardiaca en los servicios de medicina interna (estudio SEMI-IC). Med Clin (Barc) 2002; 118: 60510.

7. Suárez Herranz P, Gabarró López N, Gil Gómez J, Millán Núñez-Cortés J. Evaluación de la calidad asistencial en la insuficiencia cardiaca. An Med Interna (Madrid) 1999; 16: 553-556.

8. Permanyer Miralda G, Soriano N, Brotons C, Moral I, Pinar J, Cascant $\mathrm{P}$, et al. Características basales y determinantes de la evolución en pacientes ingresados por insuficiencia cardiaca en un hospital general. Rev Esp Cardiol 2002; 55 (6): 571-8.

9. Fonarow GC. Quality Indicators of the Management of Heart Failure in Vulnerable Elders. ACOVE. Ann Intern Med 2001; 135: 694-702.

10. Agudo de Blas P, Montoto Otero C, Contreras Murillo E, Casado Mesegué O, Conthe Gutiérrez P. An Med Interna (Madrid) 1999; 16: 557561. 